\title{
Managing Disruptive Events: How To Create Interdepartmental Responses
}

H. Richard Priesmeyer, St. Mary's University, USA

Mary Ann Murray, St. Mary's University, USA

\begin{abstract}
This article describes new concepts and methods that have been successfully applied to manage disruptive events in a large scale hospital setting. Specifically it addresses problems with service quality that emerge from disruptive events and introduces the concept of wholism as a conceptual foundation for fostering interdepartmental responses. Finally, it presents an instructional method for teaching interdepartmental interdependency in corporate or university settings.
\end{abstract}

Keywords: Disruptive Events; Quality; Interdepartmental

\section{INTRODUCTION}

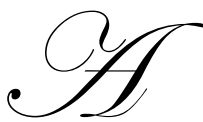

ttempts to increase service quality in an organizational setting commonly focus on increasing adherence to established standards. Quality management programs such as TQM and ISO 9000 are largely concerned with minimizing variation and increasing consistency in performance measures. These approaches to managing performance are largely successful but never entirely so. Various reasons for the shortcomings of quality management programs have been given. Such programs are typically internally focused on conformance rather than performance thereby allowing for the production of quality products and services with less than optimal demand. "Like some of the other standards, ISO 9000 fails to directly address the maintenance aspects of quality" which can easily disrupt complex, integrated manufacturing processes (Norman, 1993).

Maintenance is but one source of disruption that can affect quality. Sheffi provides a broad range of disruptive events that perturb organizational activities (Sheffi, 2005). These range from relatively minor but frequent events such as staff shortages to rare but profound disruptions such as such as severe weather and wildfires. He also provides a framework for understanding them and others have provided models for assessing the risk associated with them (Barker\& Haimes, 2009). Sheffi classifies vulnerabilities as strategic, operational, financial or hazardous and offers a classification scheme that positions disruptions on a matrix based on probability of occurrence and severity of consequences. Perhaps most useful to the current topic is the "Disruption Profile" he offers which is presented below and allows us to understand how disruptive events directly affect service quality.

\section{SERVICE QUALITY AND DISRUPTIVE EVENTS}

Our insight into disruptive events comes from a long term working relationship with a major traumatreatment hospital in the northeast. That hospital has consistently demonstrated exemplary service performance and continues to do so today largely because of their intense focus on measuring and improving service metrics. This setting, therefore, provided an ideal laboratory for implementing and then measuring the effectiveness of various quality improvement initiatives over several years.

Service quality at the hospital is defined by two primary measures: service performance and service recovery. A service performance success is said to occur when service is rendered within a specified performance threshold. For example, patient transfers permit up to a fifteen minute delay from the designated time for transfer. If a patient is to be transferred from a room at 7:00 A.M. patient escort achieves a successful service performance so long as the patient is moved from the room before 7:15 A.M. If the transfer occurs after that time then the patient escort team enters a service recovery stage that permits another 15 minutes before the recovery is considered a 
failure. An overall measure of service quality was derived as a weighted average of these two measures giving full weight to service performance successes and half weight to service recovery successes. These measures, systematically collected throughout the hospital, provide rich metrics of service quality at the hospital. The two dimensions of service quality apply throughout the institution to a wide range of operations ranging from patient transport times to phlebotomy to the accuracy of prescriptions distributed by the pharmacy.

Initial efforts at improving service quality at the hospital focused on improving the effectiveness of the teams responsible for managing activities in the various functional areas of the hospital. Improvements in quality control reports and changes in the memberships of the teams effectively increased overall quality measures above the 90 percent rate. This level of service performance is well above industry standards which average at the mid to low 80 percent rate for comparable measures.

It was in an attempt to push performance over the 95 percent service quality that we learned the limits of these initial attempts and were forced to explore other causes of service failure. Our search led us to the importance of disruptive events and, subsequently, to a novel approach to managing service quality.

Figure 1 provides the Disruption Profile for an actual event- a hospital fire- that occurred in the basement of the hospital. The event relates to a fire in a flatwork ironer in the textile department. This operation processes more than a million pounds of laundry per month to support hospital operations. The flatwork ironer is a large machine some thirty feet long that dries, presses, and folds laundry in seconds; it is instrumental in the processing of a continuous flow of textiles.

Disruptive events are typically preceded by a set of conditions that allow them to occur. These conditions may or may not be knowable or avoidable. Early indicators of a disruptive event are typically ignored and, when they are ignored, the probability of the event increases (Sheffi, 65). In the case of the textile fire these early indicators were (1) old equipment, (2) high demand, and (3) construction in the area. Ironically, the construction was to make way for a new flatwork ironer to replace the one that caught fire. Figure 1 provides the events associated with the fire and relates them to the common profile for disruptions.

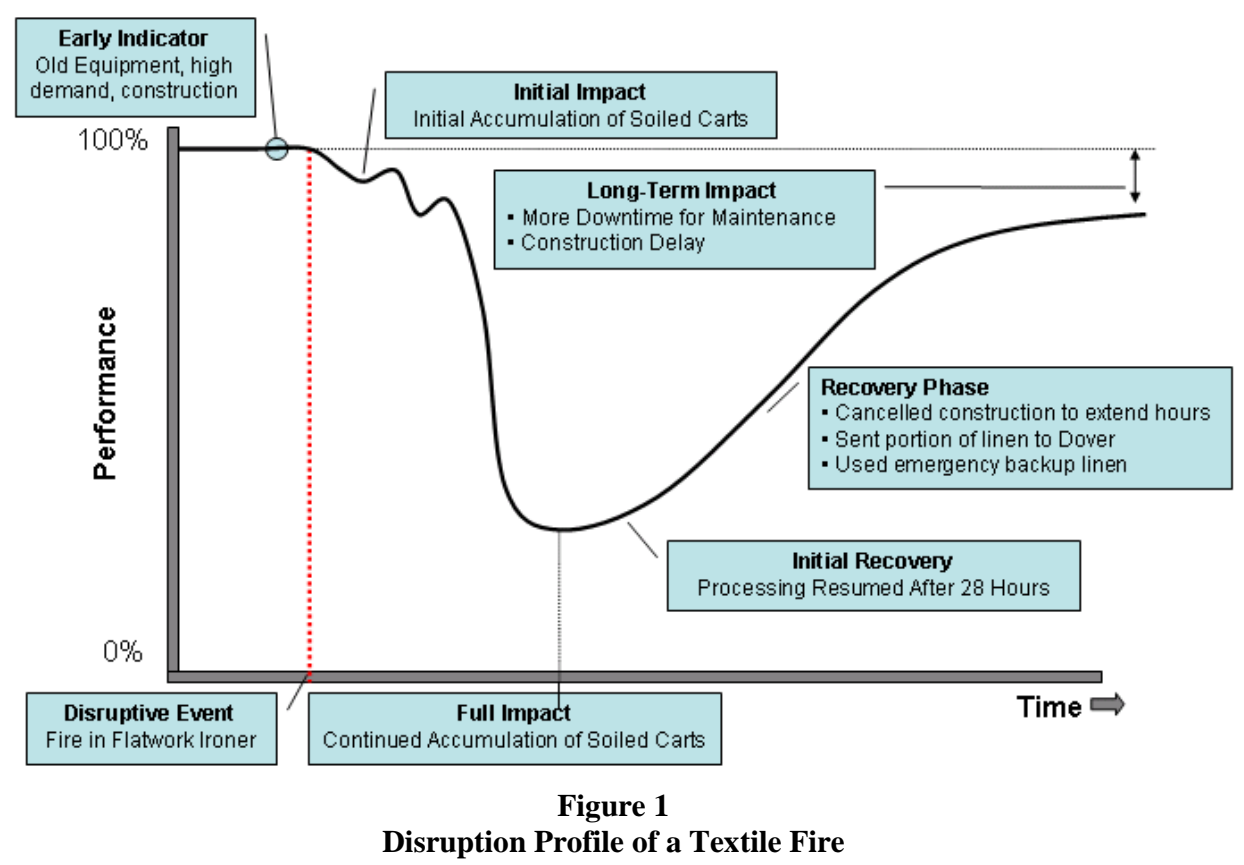


Of particular interest here is the long term impact if this disruption. Additional downtime for the machine, necessary to prevent a similar event, and construction delays caused by the event resulted in a protracted reduction in overall performance in the textile department. And the effect of the disruption is not limited to textiles, it propagates throughout the hospital effecting nursing, environmental service, the emergency department and even admissions should the number of available beds be affected. Even though the department may return to full performance the effect of the event is clear; it serves to reduce performance throughout the hospital and, once recovered, it is likely replaced by another disruptive event with widespread impacts on service quality and another protracted recovery stage. Repeated disruptive events, however severe and irregular, suppress performance and prevent improvements in service performance beyond some threshold; perhaps that threshold is 95 percent.

\section{THE CONCEPT OF WHOLENESS}

The term "wholeness" has emerged to describe a new view of complex systems beyond that offered by traditional systems theory. Rather than focusing on the parts of a system, a wholeness view defines parts as only artifacts of a larger whole. The whole is considered primary and the parts secondary with emphasis on understanding the whole. The wholeness approach recognizes the existence of a system-wide culture that ultimately relies on individual members to organize their behavior. This observation was also identified by Edgar Schein $(1999,14)$ when he stated, "Culture exists at the level of the whole organization if there is sufficient shared history. It is even found at the level of a whole industry because of the shared occupational backgrounds of the people industry-wide". Compared to more traditional views, wholeness suggests changes in how one should communicate and manage in a complex organization (Bohm, 1996). Rather than viewing the organization as "independent parts" the wholeness view calls for it to be managed as one "interdependent whole." The responsibilities for problem solving and decision-making shift from individual system members to all system members collectively. Table 1 compares the traditional view to the wholeness view with respect to these and other important management attributes.

Table 1

Comparison of Traditional and Wholeness Management Views

\begin{tabular}{|l|l|l|}
\hline & \multicolumn{1}{|c|}{ Traditional View } \\
\hline Perspective & Independent and part-centric & Wholeness View \\
\hline Decision-Making & Single member & Shterdependent and wholistic \\
\hline Space and Time & Local and immediate & Totality and continuous \\
\hline Leadership & Command and control & Facilitate, teach, and create \\
\hline Communications & Linear exchange & Openness and receptiveness \\
\hline Measurement & Single variables over time & Multiple, dynamic, interactive \\
\hline Effectiveness & Departmentally specific & Throughout the whole \\
\hline Learning & Periodic and skill based & Continuous, relation based \\
\hline
\end{tabular}

The wholeness view draws attention to the interdependencies among the parts and thereby changes the focus of decision-making. Problems are viewed as problems of the whole rather than associated with any single part. Solutions, likewise, emerge from a better understanding of the whole. The organization is managed in its totality rather than as autonomous parts with immediate concerns. Those responsible for managing activities assume new roles. Managers facilitate the open exchange of ideas, teach, and seek creative solutions rather than attempt to control the system by authority or policy. Communication among departmental managers takes on an entirely new form. Rather than merely an exchange between individual members, communication takes place in sessions that foster greater understanding of the entire system. Likewise, new techniques for measurement such as metrics that capture the relationship of service performances in different departments are used to capture the interactive and dynamic behavior of the system. The wholeness view and the methods that support it can produce improved efficiencies throughout the system and, because participants continuously learn more about the changing behavior of the system, a new culture of cooperation and mutual benefit can emerge. This change in culture has been linked to reduced turnover among administrators in hospitals (Hwang \& Chang, 2009) and can cause improvements to be sustained without repeated initiatives and renewed efforts. Most importantly, when employees throughout an organization adopt a wholeness view they find numerous creative ways to assist departments with disruptive events that would never otherwise be discovered. 
As promising as the concept of wholeness is, it differs so much from traditional views and requires so many individuals to rethink their roles in the organization that it seems quite impossible to apply. Yet we were able to use the concept of wholeness to foster hospital-wide responses to managing disruptive events. We did this by taking a very practical approach to discovering the interdependencies that exist between functional departments in the hospital then using a powerful analogy that convincingly demonstrated the need for interdepartmental responses to operational disruptions.

\section{IDENTIFYING DEPARTMENTAL INTERDEPENDENCIES}

Besides measuring the performance of the individual functional departments in the hospital, our service performance and service recovery measures could also provide objective measures of the interdependencies that exist between departments. We began our exploration of departmental interdependencies by quantitatively examining the relationships between the departmental service quality measures throughout the hospital. Specifically, we looked for correlations in the performance measures and used the results in a series of workshops with departmental managers to begin a discussion of interdepartmental dependencies.

It became clear that the statistical measures failed to capture many of the more subtle but critical interdependencies. These additional interdependencies were revealed in a workshop in which departmental managers completed an interdependency matrix as shown in Table 2.

Table 2

Departmental Interdependency Matrix

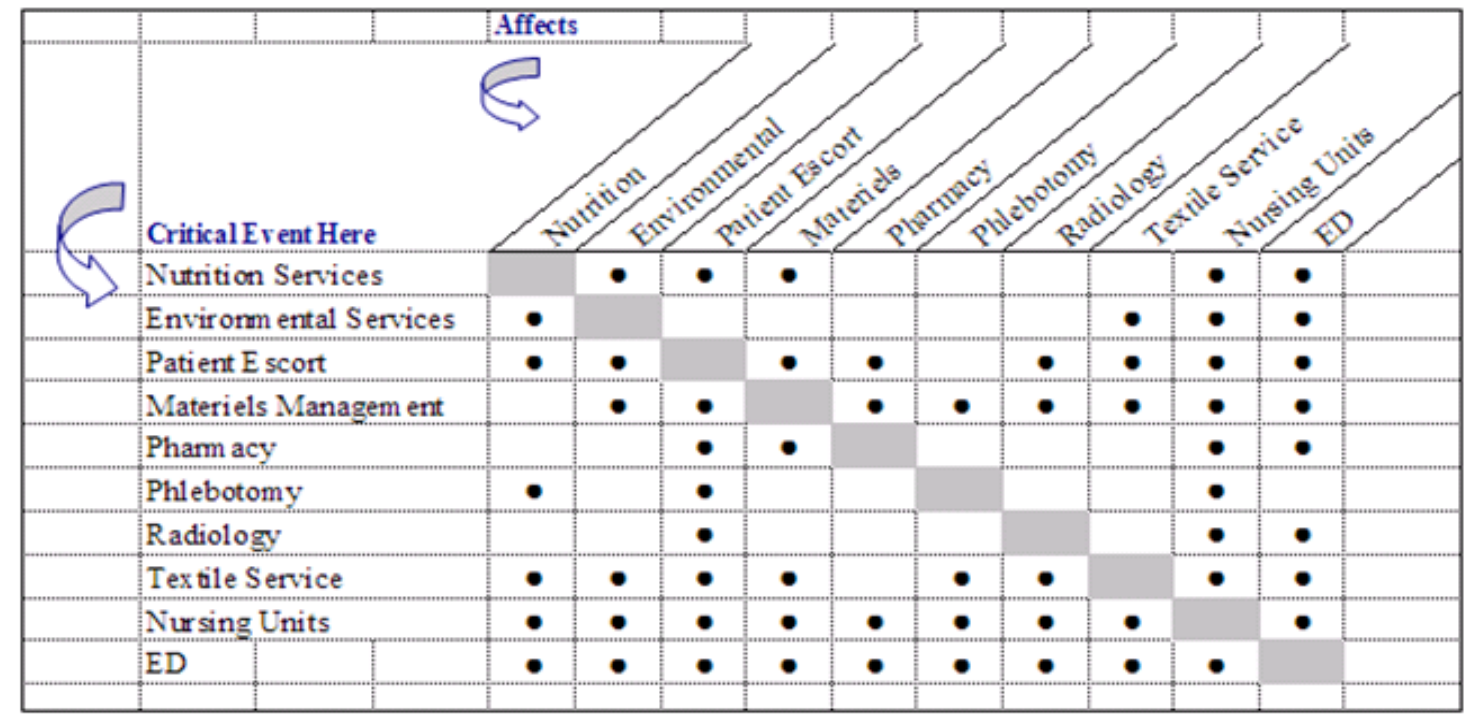

Departmental managers were asked to identify a disruptive event within his or her department that might cause a significant reduction in service performance. Each manager then described the event to the other departmental managers and asked what affects, if any, would result from the disruption. Those managers that identified significant impacts on their own operations noted the relationship on the matrix. Proceeding through each department, the management team completed the matrix and, in doing so, mapped the dominant interdepartmental dependencies that exist in the hospital. This exercise revealed the sensitivity of the hospital system to various types of disruptions. Specifically, it indicated which events could be accommodated more easily by the system and which tended to overwhelm the system and required a system-wide response (Gholz \& Press, 2010). 
Examples of disruptive events cited by the managers ranged from temporary staffing shortages to surges in demand in the Emergency Department (ED) and equipment failures in Radiology. It is interesting to note on the matrix the number of departments dependent on the performance of the Textiles department.

\section{THE WHOLENESS ANALOGY}

Knowing that departmental interdependencies exist does not create a mindset that one is part of a single, undivided whole much less the motivation to voluntarily take action to assist others when they are challenged with a disruptive event. A model of the system, whether abstract or quantitative, can offer valuable insight into how disruptive events can be managed (Janic, 2009). The part-centric perspective that causes employees to take care of their own and let others fend for themselves is a fundamental characteristic of most organizations. Changing that view to a new perspective of mutual interdependency requires a new mental model which can be created with an effective analogy.

We settled on an island chain for our wholeness analogy and mapped the functional departments of the hospital onto a satellite photo of the Greek islands. Replacing the island names with pseudonyms for each department we produced an image that captures both the part-centric view of separate operations in the hospital and the wholistic perspective of a shared "Interdependent Sea" (see Figure 2). The creativity of this approach offered a fresh perspective of the issue of interdependency while still being grounded in the practical reality that separate departments do exist.

Building on this image we developed a computer program that incorporated the Interdependency Matrix in Table 2. Clicking on any island in the Interdependent Sea would simulate a disruptive event in that department and highlight those other islands (i.e., departments) that would be affected by that event. Figure 2 depicts a disruptive event in Textiles.

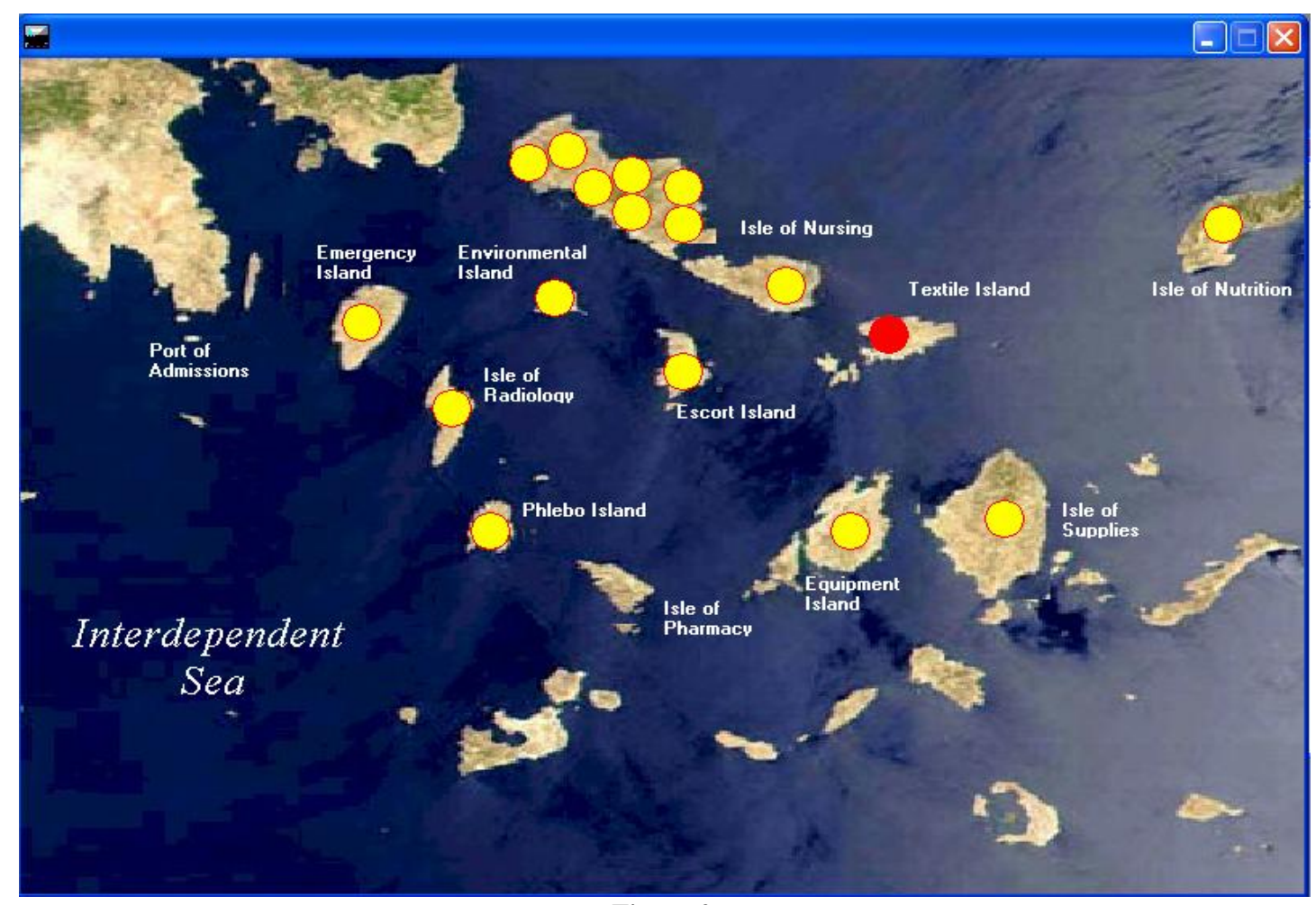

Figure 2

The Interdependent Sea 


\section{DEVELOPING RESPONSE STRATEGIES}

The Interdependent Sea program provided the basis for simulating disruptive events anywhere in the hospital. In a workshop setting with departmental managers we solicited examples of specific disruptive events then modeled them with our program. To foster thinking about how to respond to each event we asked all managers what action they might take to help mitigate or otherwise assist in managing the event. Note that their assistance might be directed toward the department with the event or directed toward one of the departments affected by the event.

These simulations resulted in numerous creative intervention strategies for any given event. Patient escort identified ways to help manage equipment shortages. Nursing identified ways to help nutrition deal with staff shortages. Nutrition identified ways to help the Emergency Department handle surges in patient load. The response strategies were so many, so varied, and so easy to produce that it was decided they should not be captured in any training document but rather generated when needed.

\section{COMMUNICATING THE NEED}

Managing disruptive events requires a way to recognize that such an event is occurring and a means of communicating that fact to others likely to be affected by it. We addressed both of these issues in a rather straightforward way. Guided by the knowledge that disruptive events typically have early indicators we asked each department manager to establish a threshold value that would signal a likely disruptive event in the department. For example, nursing established a specific threshold for the patient/staffing ratio that included a measure of patient acuity. Beyond this threshold the nursing department would alert others of a disruptive event in nursing. Textiles established a limit to the backlog of unprocessed linen carts beyond which they would alert others of a disruptive event in Textiles. The Emergency Department established a threshold for visits per hour beyond which they would signal others of their event.

The signaling of these events was facilitated by a paging network that was based on the Departmental Interdependency Matrix in Table 2. To signal an event any departmental manager could call the hospital operator and simply state they have a "service alert". To this they could add a short phrase to describe the nature of the event. The hotel operator would then simple transmit the alert through the paging network which would notify the departmental managers in those affected departments.

All service alerts were logged and reviewed by departmental managers each month. During these reviews managers were asked to describe what actions they took to support the department signaling the alert. As expected, there was some initial reticence to signal alerts at first and some resistance to respond to the alert system. While it has not been determined whether this approach will allow us to move beyond the 95 percent threshold for service quality that we seek some early successes have demonstrated that it does minimize service interruptions and it has, therefore, been established it as an ongoing quality management initiative.

\section{HOW TO TEACH DEPARTMENTAL INTERDEPENDENCY}

The success at fostering a wholistic view and an understanding of departmental interdependency in a corporate setting suggested that these concepts can be taught. The appropriate setting for this topic at a university is in the capstone strategic management class which seeks to integrate content from functional topics such as marketing, human resources, finance, production, and information systems. Below is a team-based approach that has been used repeatedly with considerable success. It is an engaging exercise for students and it provides a variety of results that are interesting when reviewed by the entire class.

This exercise can be conducted in two to three class meetings with some out-of-class time dedicated to refining and finalizing the report. Much of the work can be conducted in class with students using on-line resources and individual laptops. The exercise is very much a process of discovery with the instructor directing each step of the analysis not revealing the overall exercise in advance. In keeping with this approach, the instructions below are provided in much the same way as they would be given to the students. The final "deliverable" is a PowerPoint presentation from each team. 


\section{Select and Define a Company}

Begin by establishing teams of about four students each and have teams identify a central subject for analysis. Specifically, have teams select a company of their own choosing to which they will apply the exercise. The key is to be able to identify multiple operational activities within the firm. Photography studios, large box retailers, and small manufacturers have all worked well. An example of how this exercise was applied to a regional winery will be provided. After selected the firm students should capture an image of the firm and use it as an opening slide to their PowerPoint presentation.

Once selected, students should define the major functional departments of that firm and describe some of the specific activities in each of those departments. Some of this information will be available on line; however, students should be able to anticipate what primary activities would exist should that information not be immediately available. This content should be incorporated into the presentation. The winery application identified event planning, restaurant food service, wine distribution, wine production, and sales as primary centers of activity. Students described various activities likely to occur within each of these centers.

\section{Develop the Departmental Interdependency Matrix}

Students should develop an interdependency matrix that relates the functional activity areas (i.e., departments) to each other. The matrix provided in this article can serve as an example. They should then consider how these activity areas interact. The matrix can be developed in Excel or as a table and placed into the presentation. It is useful to have students document the nature of the interdependencies they identify so these can be presented as examples in the completed project. The team working on the winery application cited seventeen important interdependencies among the five centers of activity and recorded a description of each.

\section{Create an Interdependent Sea}

Instruct students to find a satellite photo of an island chain. You may wish to provide the example in this article to help describe the type of image you are asking them to find. The key is to find a map of multiple separate islands with minimal text in the image. Once located, have students map their departments of functional activity onto the island map. All of the activities described above may be accomplished in a single class leaving students to refine their maps after class. Once completed, two copies of the island map should be incorporated into the students' presentation. The student map for a winery appears in Figure 3.

\section{Create and Respond to Disruptive Event Scenarios}

Students should now be asked to consider two or three scenarios in which a major disruptive event occurs and affects their chosen company. For each of these scenarios they should describe activities which require other departments to assist in managing the event.

The Interdependency Matrix should be used to guide their suggestions. In the winery example students created scenarios for the following: (1) a freeze that destroyed 65 percent of the grape harvest, (2) an accidental double booking of event facilities and, (3) a major theft from the distribution warehouse. The departmental strategies created to manage the disruptive event should be documented and placed into the presentation. These can either be in table form or mapped directly onto the island map. Figure 3 provides the intervention strategies for the winery example. 


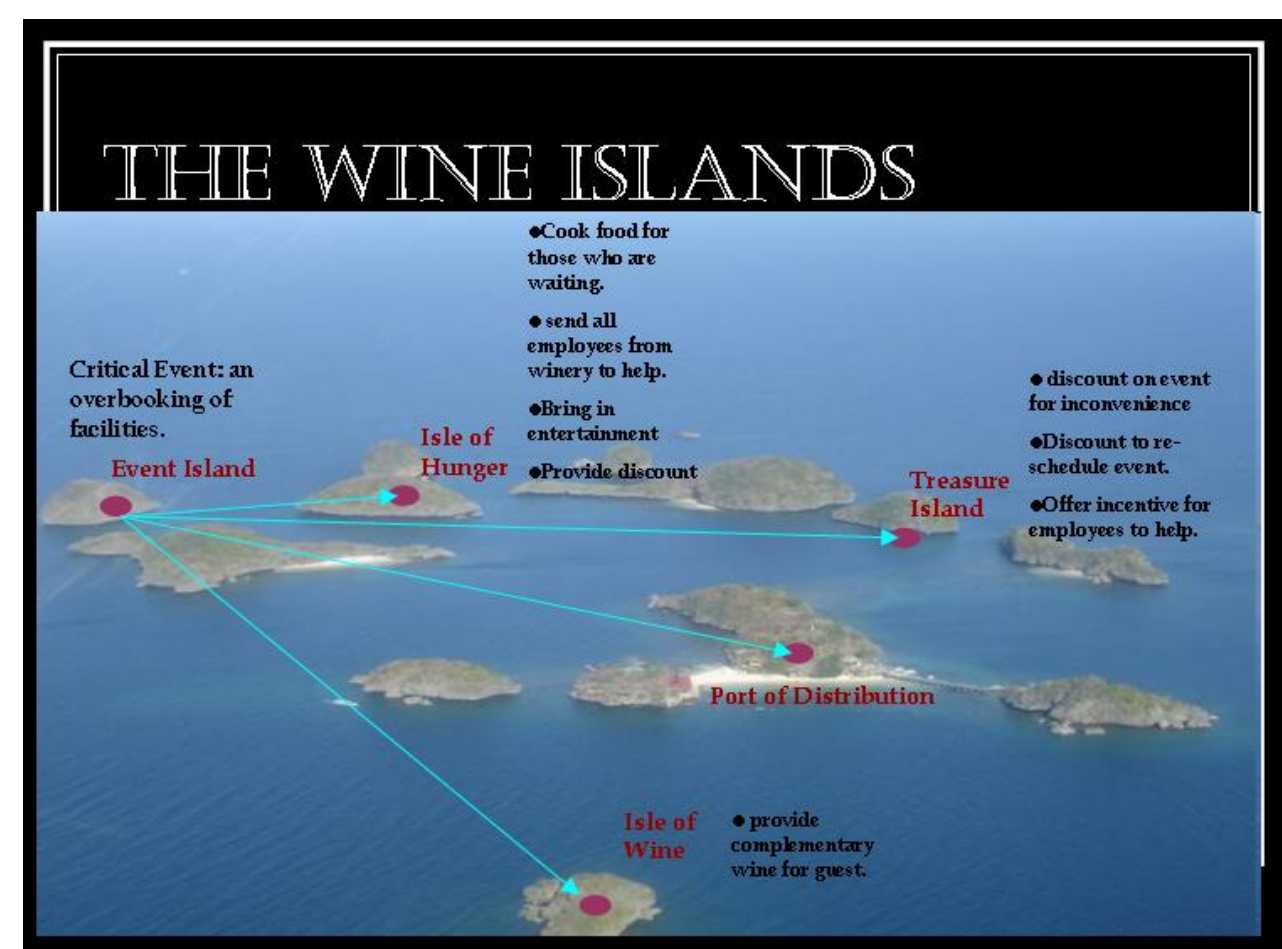

Figure 3

Student Map of an Interdependent Sea with Scenario

\section{Present and Discuss Disruptive Events and Interdepartmental Responses}

As each student team presents one of their scenarios, seek to identify some of the more creative strategies they have identified to manage the disruptive event. Emphasize the opportunity that departments have to be proactive in their response rather than waiting for instructions from some central authority. Most importantly, use the analogy of dependent islands to create an understanding of shared interdependency and overcome the tendency for traditional, part-centric thinking.

\section{CONCLUSION}

Out attempts to improve service quality in a complex healthcare setting identified disruptive events as a source of irregular but continued interruptions which reduced service performance. Attempts to mitigate these disruptions called for immediate interdepartmental responses. We discovered that the concept of wholeness provides the conceptual foundation and that such responses can be encouraged through workshop training sessions that built upon our Interdependent Sea analogy. This analogy made it easy for departmental managers to offer creative intervention strategies for any department suffering a service interruption. We implemented the program with a pager system at the hospital.

We also have confirmed that the concept of interdependency and the need for departments to work together can be taught in a college setting and we have included pedagogy for teaching interdepartmental responses in strategic management classes. 


\section{AUTHOR INFORMATION}

H. Richard Priesmeyer, Ph.D. is the Emil C. Jurica Professor of Management and chair of the Department of Management in the Bill Greehey School of Business at St. Mary's University. His research has focused on the practical applications of nonlinear systems theory which allow for the control and management of complex and unpredictable systems. He has been a consultant to healthcare organizations for a number of years and is author of the book "Organizations and Chaos: Defining the Methods of Nonlinear Management." E-mail: rpriesmeyer@stmarytx.edu. Corresponding author.

Mary Ann Murray, Ph.D. is associate dean in the Bill Greehey School of Business at St. Mary's University. Prior to joining St. Mary's University, Murray taught as a faculty member in the School of Business at John Carroll University. Murray's current research interests are in TQM applications and nonlinear applications to health care and education. She has been actively involved in many professional associations, including Decision Sciences Institute and the American Production and Inventory Control Society. E-mail: mmurray @ stmarytx.edu

\section{REFERENCES}

1. Barker, Kash and Yacov Y. Haimes, "Assessing uncertainty in extreme events: Applications to risk-based decision making in interdependent infrastructure sectors," Reliability Engineering \& System Safety, Vol. 94, Issue 4, April 2009, p819-829.

2. Bohm, D., On Dialogue, Routledge, London, 1996.

3. Gholz, Eugine \& Daryl g. Press, "Protecting 'The Prize': Oil and the U.S. National Interest," Security Studies, Vol. 19, Issue 3, July-September 2010, p453-485.

4. Hwang, J.I. \& H. Chang, "Work climate perception and turnover intention among Korean hospital staff," International Nursing Review, Vol. 56, Issue 1, March 2009, p73-80.

5. Janic, Milan, "Modeling Airport Operations Affected by a Large-Scale Disruption," Journal of Transportation Engineering, Vol. 135, Issue 4, April 2009, p206-216.

6. $\quad$ Norman, Richard M., "Maintenance’s Role in ISO 9000," Modern Casting, May, 1993, pp. 1-2.

7. Schein, E. H., The Corporate Culture Survival Guide, Jossey-Bass Publishers, San Francisco, 1999.

8. Sheffi, Yossi, The Resilient Enterprise: Overcoming Vulnerability for Competitive Advantage, The MIT Press, 2005. 
NOTES 